Supporting Information

\title{
Metallized Skeleton of Polymer Foam Based on Metal-Organic Decomposition for High-Performance EMI Shielding
}

Si-Yuan Liao ${ }^{1,2, \#, \text { Gang Li }}{ }^{1, \#}$, Xiao-Yun Wang ${ }^{1,2, \#}$,Yan-Jun Wan ${ }^{1, *}$, Peng-Li Zhu ${ }^{1, *}$, You-Gen $\mathrm{Hu}^{1}$, Tao Zhao ${ }^{1}$, Rong Sun ${ }^{1}$, Ching-Ping Wong ${ }^{3}$

${ }^{1}$ Shenzhen Institute of Advanced Electronic Materials, Shenzhen Institutes of Advanced Technology, Chinese Academy of Sciences, Shenzhen 518055, China

${ }^{2}$ University of Chinese Academy of Sciences, Beijing 100049, China

${ }^{3}$ School of Materials Science and Engineering, Georgia Institute of Technology, Atlanta 30332, United States

\# These authors contributed to the work equally

*E-mail: yj.wan@siat.ac.cn (Y.-J Wan), pl.zhu@siat.ac.cn (P.-L. Zhu) 
The volume fractions of silver nanoparticles in the Ag@ME are calculated to be 0.89 vol.\%, 2.03 vol. \%, 2.58 vol. \% and 3.48 vol\% accorting to the Eq.(S-1, 2):

$$
\begin{gathered}
W_{A g}=W_{A g @ M E}-W_{M E} \\
V_{A g}=\frac{w_{A g} / \rho_{A g}}{V_{A g @ M E}}
\end{gathered}
$$
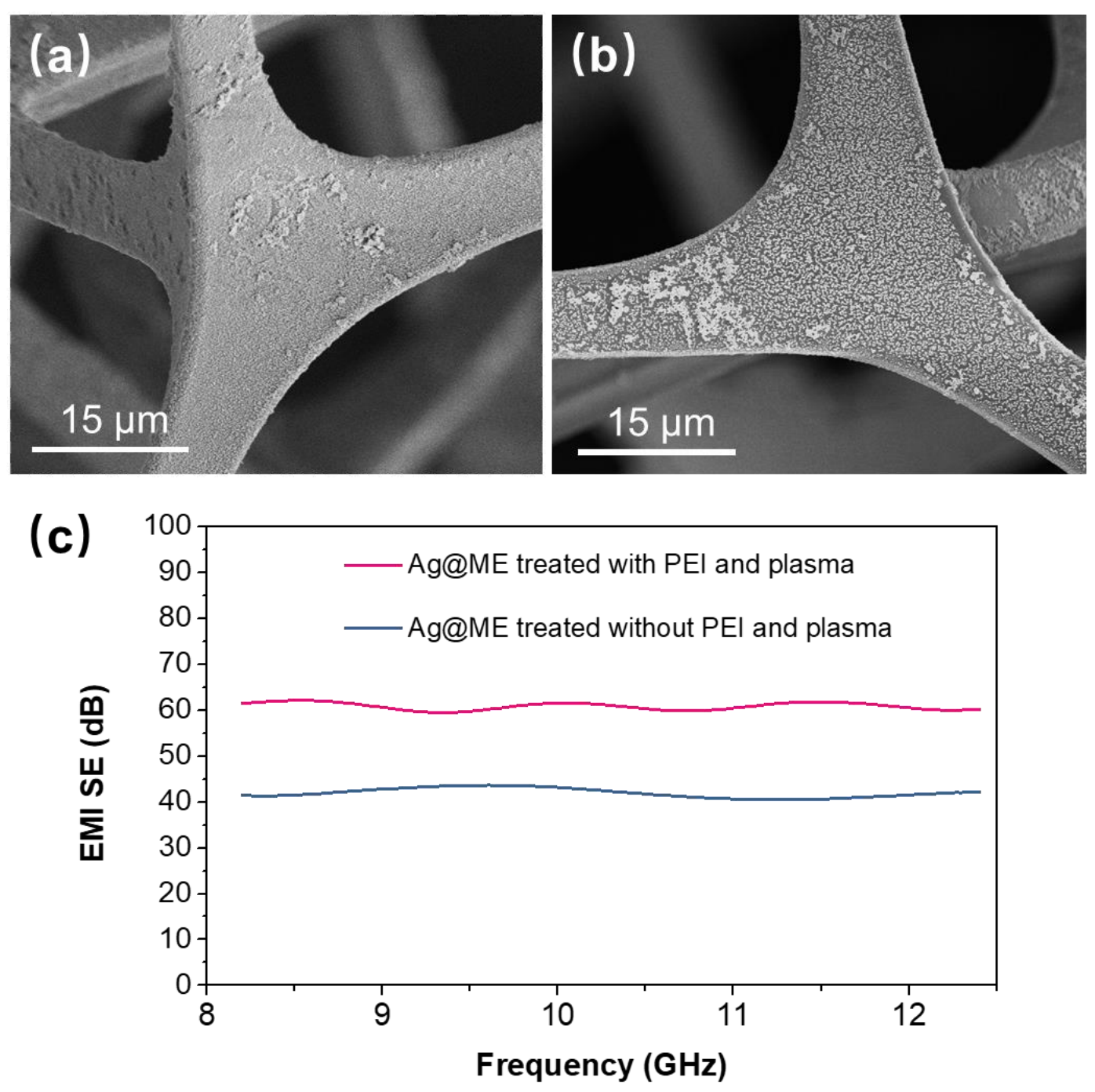

Figure S1. Morphology of silver deposited on ME skeleton of (a) with PEI and plasma treatment and (b) without PEI and plasma treatment; (c) EMI shielding performance of Ag@ME treated with and without of PEI and plasma at thickness of $\sim 5 \mathrm{~mm}$. 

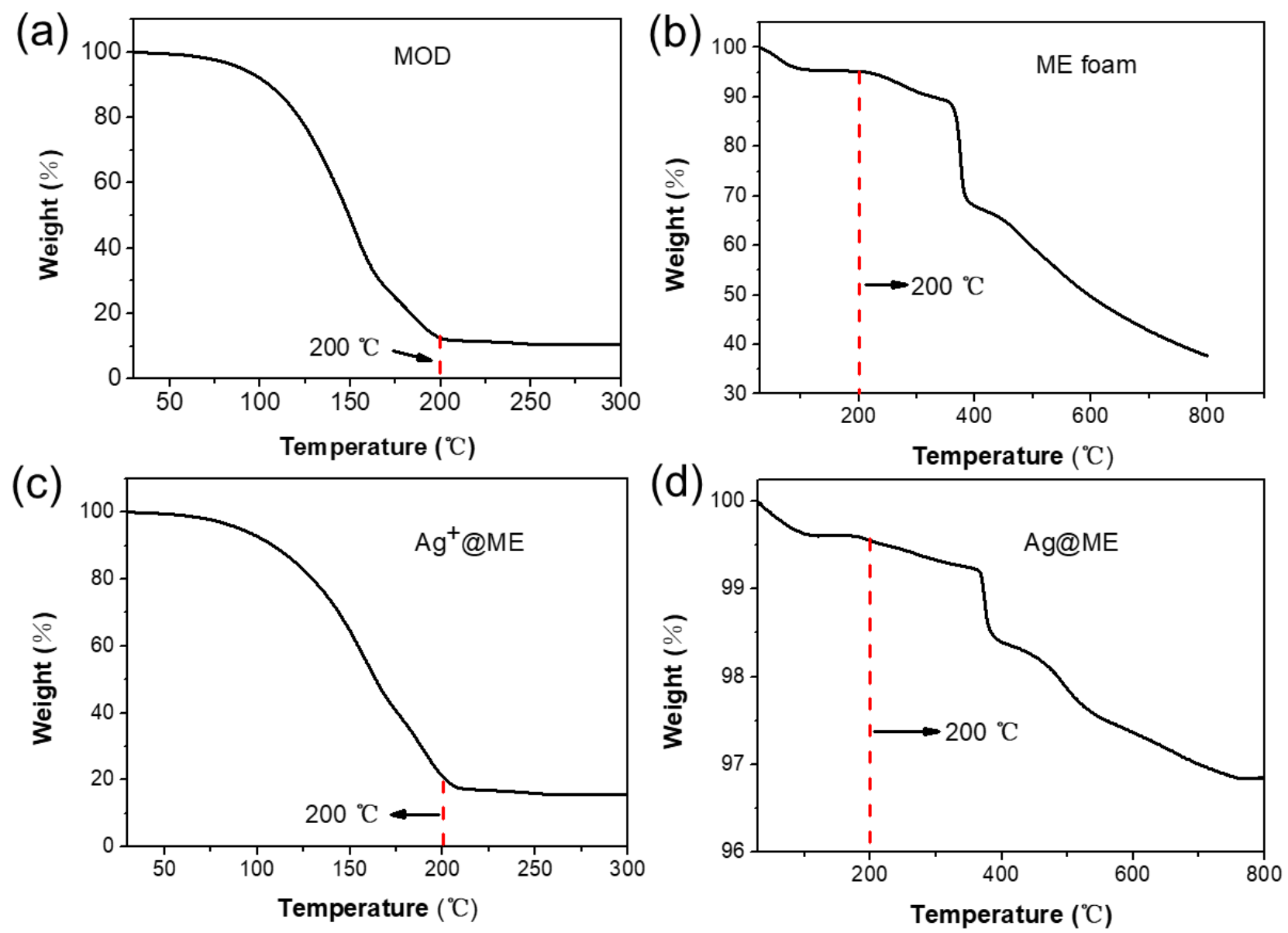

Figure S2. Thermogravimetric analysis curves of (a) MOD, (b) original ME foam, (c) $\mathrm{Ag}^{+} @ \mathrm{ME}$ and (d) Ag@ME. 

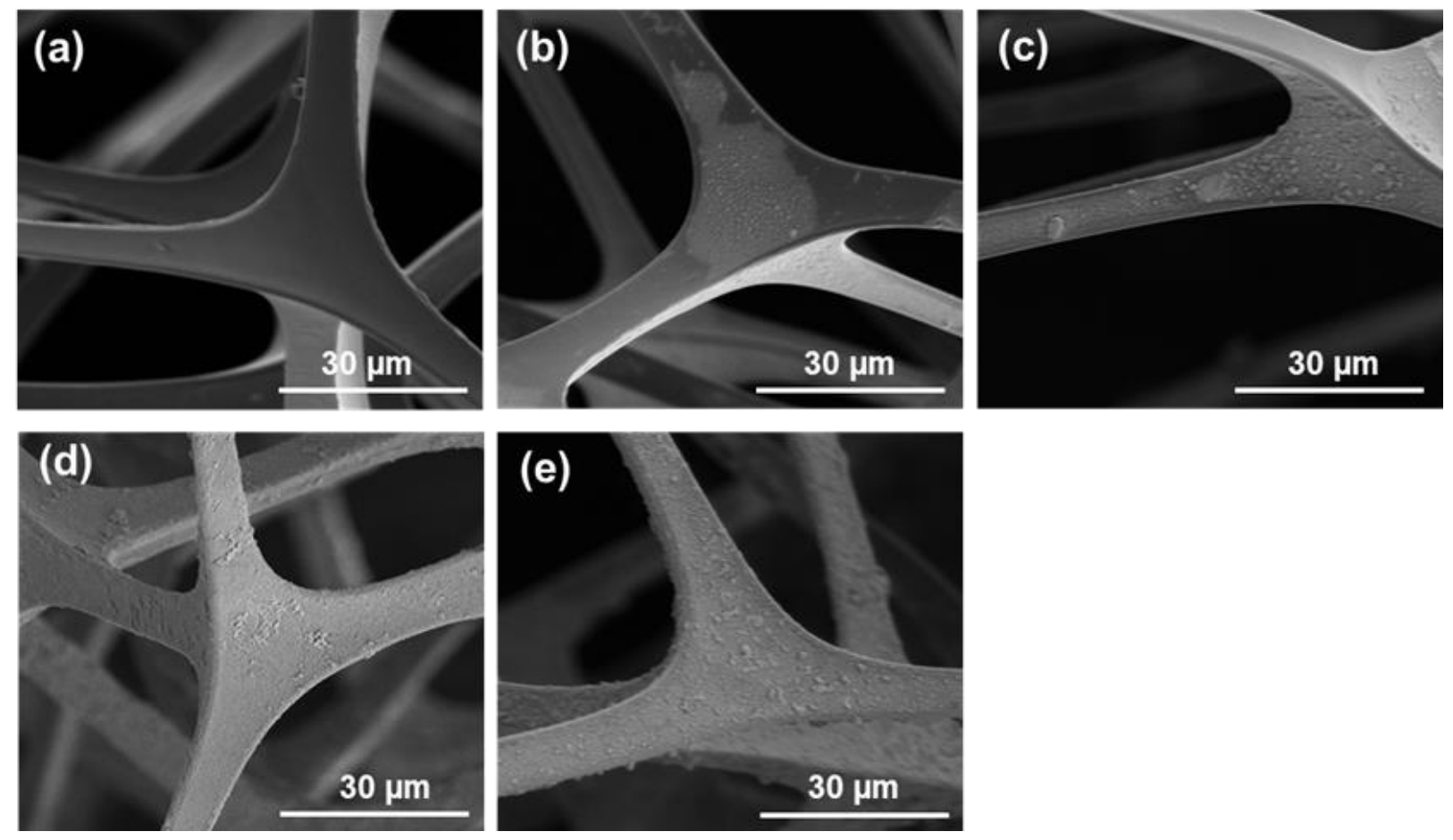

Figure S3. SEM images of sintered Ag@ME with different soak time in MOD: (a) 0 hour, (b) 4 hours, (c) 8 hours, (d) 12 hours and (e) 24 hours.
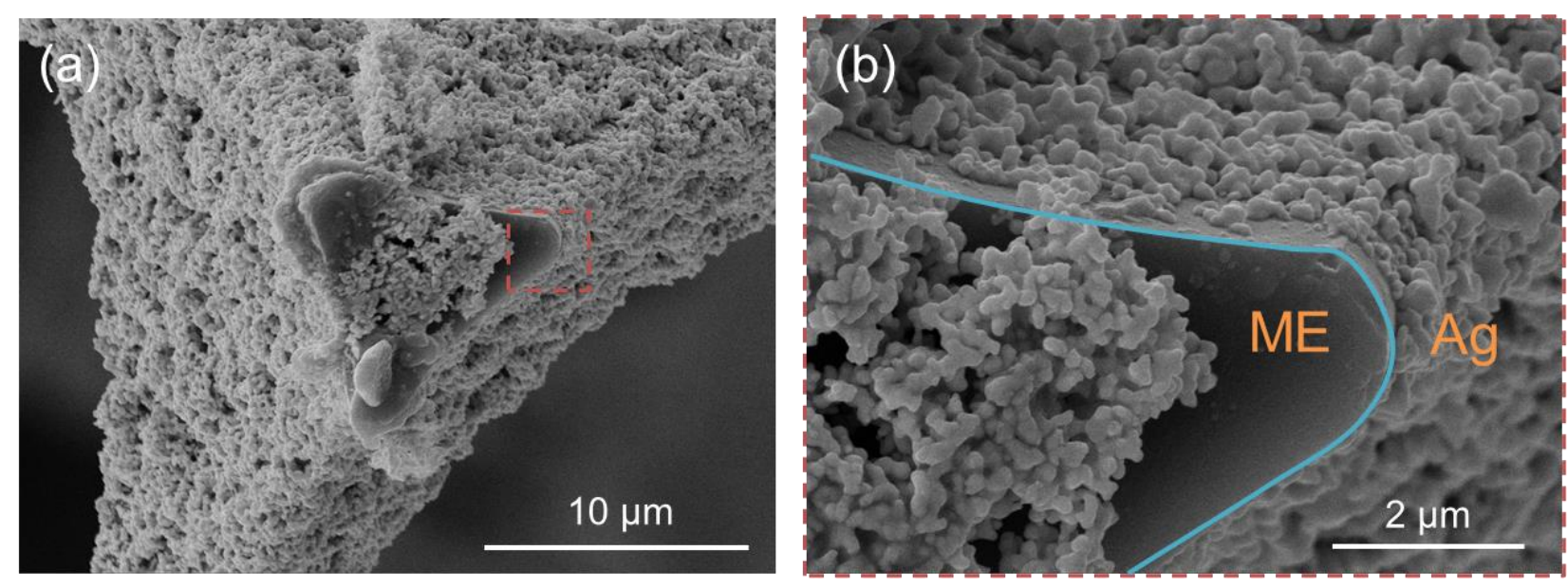

Figure S4. (a, b) SEM images of cross-sectional ME skeleton after deposition of Ag. 

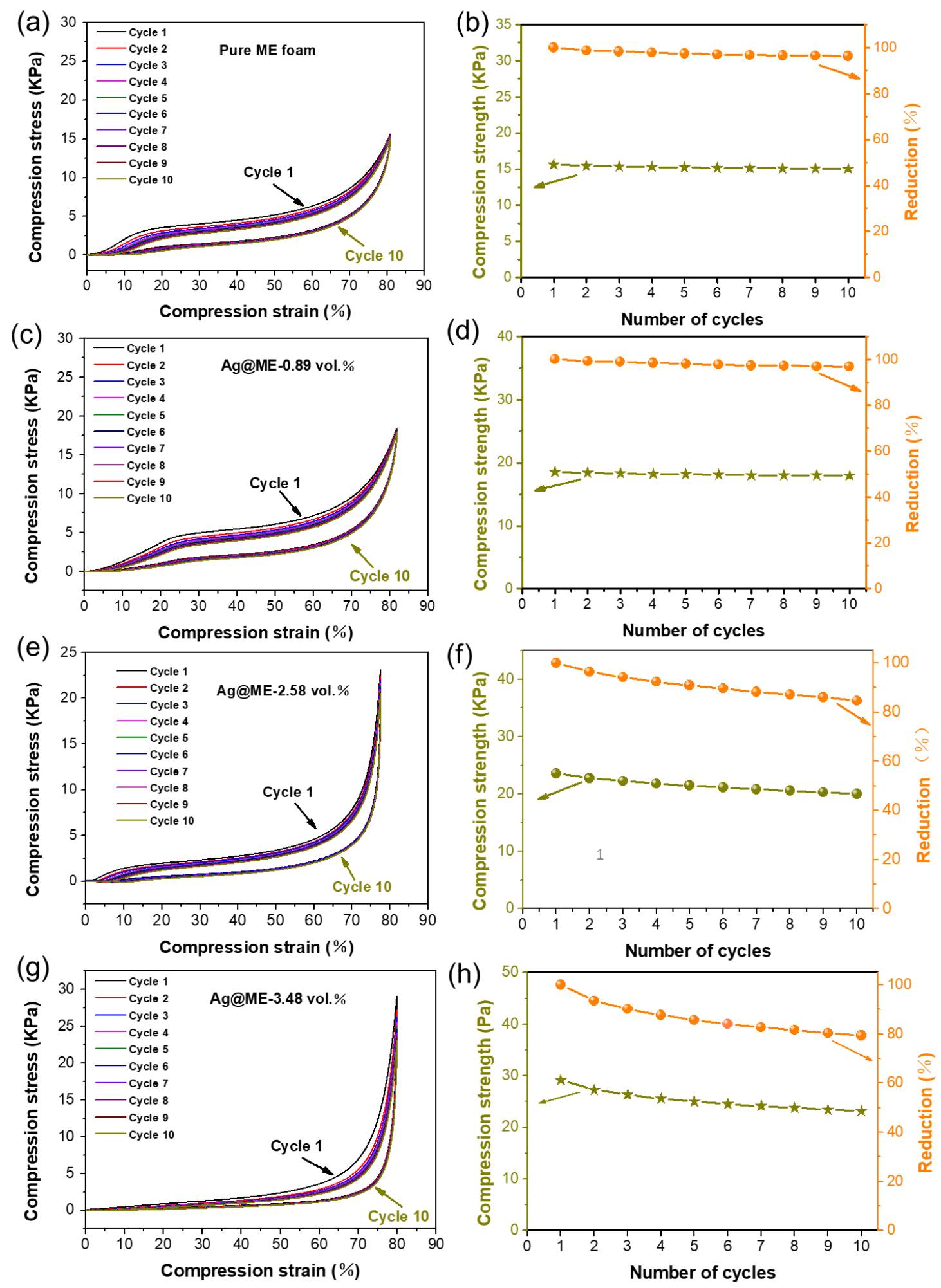

Figure S5. (a, c, e, g) Stress-strain curves of different sample with $80 \%$ compression ratio during loading-unloading cycles. (b, d, f, h) Statistics of relationship between compression strength and reduced ratio under loading-unloading cycles with $80 \%$ compression strain. 

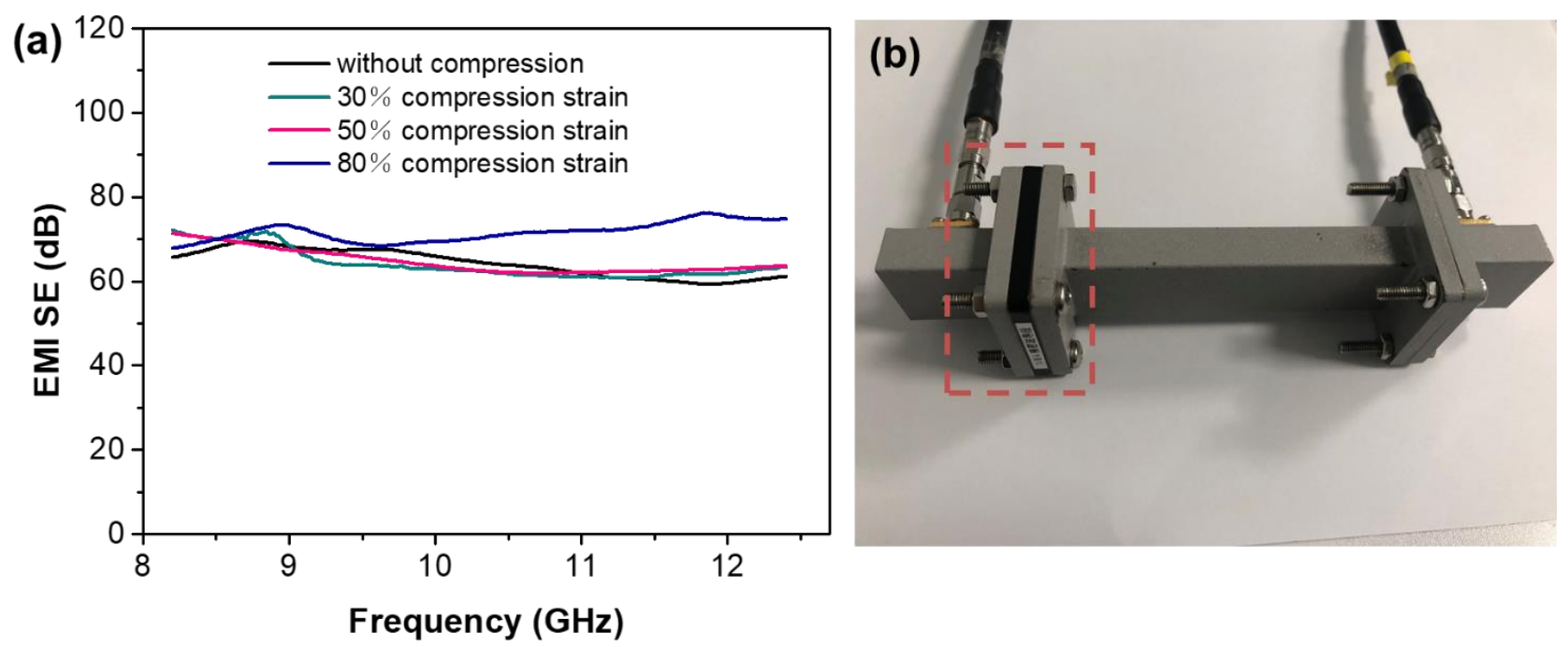

Figure S6. (a) EMI SE of Ag@ME-2.03 vol.\% with different compression strains (0\%, 30\%, $50 \%, \sim 80 \%$ ), the original thickness of the sample is $\sim 5.8 \mathrm{~mm}$. (b)The compressed sample is placed at different fixture with corresponding thickness to measure the EMI SE under compression, in which the left and right sides of compressed sample are fixed with PET membrane. 
All Electromagnetic simulation work in this article was carried out through software. All simulation results are obtained based on the frequency domain solution workflow. For the rectangular waveguide fixture, the size is set to $40 \times 22.86 \times 10.16,40 \times 15.80 \times 7.90,40 \times 10.67 \times 4.32$ and $40 \times 7.12 \times 3.56$ $\mathrm{mm}^{3}(x \times y \times z)$ correspond to $\mathrm{X}-, \mathrm{Ku}-, \mathrm{K}-$ and Ka-band, respectively. The material is set to PEC (perfect electric conductor). The $\mathrm{S} 1$ is the emission port of the excitation source where the excitation type is gaussian excitation, and S2 is received port of transmitted electromagnetic wave. The electromagnetic wave transmission mode is the standard $\mathrm{TE}_{10}$ transmission mode in the rectangular waveguide transmission model, wherein the YZ plane of the cuboid is selected as the excitation face of the wave port and electromagnetic wave propagation along the $\mathrm{X}$ direction. The electrical conductivities of Ag@ME-0.89vol.\%,Ag@ME-2.03 vol.\%,Ag@ME-2.58 vol.\% and Ag@ME-3.48 vol.\% foam are set as 1.2 S/m, 158.4 S/m, 198.3 S/m and 279.2 S/m, respectively, which was obtained from the previous conductivity experimental results.

Table S1 EMI simulation parameter of Ag@ME

\begin{tabular}{|c|c|c|c|c|}
\hline Parameters/Sample & 0.89 vol.\% & 2.03 vol. $\%$ & 2.58 vol. \% & 3.48 vol. \% \\
\hline Conductivity (S/m) & 1.2 & 158.4 & 198.3 & 279.2 \\
\hline Permeability & \multicolumn{4}{|c|}{1} \\
\hline Length (mm) & \multicolumn{4}{|c|}{ The size is consistent with the rectangular waveguide } \\
\hline Width (mm) & \multicolumn{4}{|c|}{ The size is consistent with the rectangular waveguide } \\
\hline Model boundary & \multicolumn{4}{|c|}{ Perfect electric conductor } \\
\hline Mesh quality & \multicolumn{4}{|c|}{ Default } \\
\hline Frequency (GHz) & \multicolumn{4}{|c|}{$8.2-40$} \\
\hline Excitation type & \multicolumn{4}{|c|}{ Gaussian excitation } \\
\hline
\end{tabular}




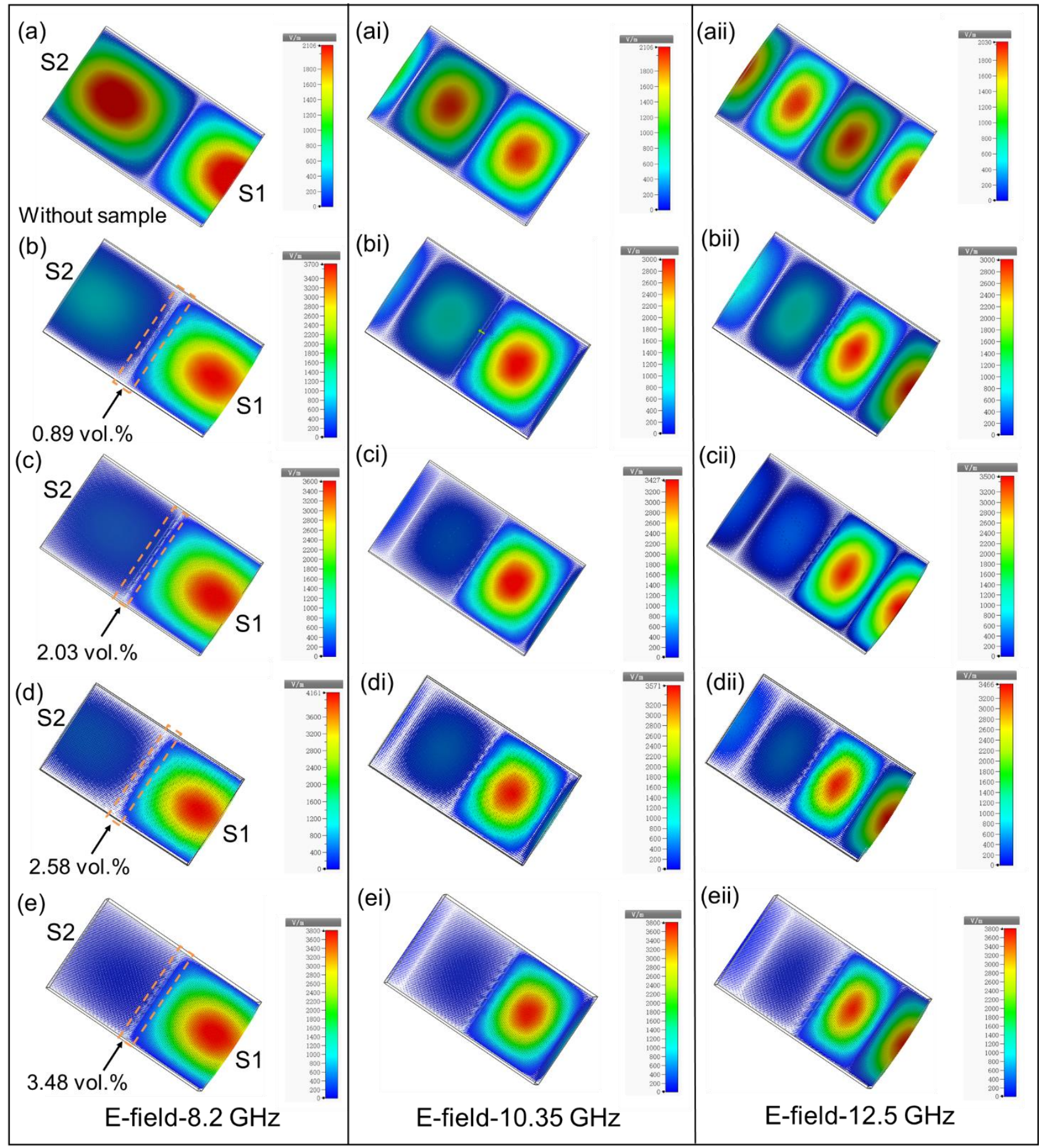

Figure S7. Electromagnetic simulation results obtained by software. Electric-field intensity distribution of (a) without sample, (b) Ag@ME-0.89vol.\%, (c) Ag@ME-2.03 vol.\%, (d) Ag@ME2.58 vol.\%, (e) Ag@ME-3.48 vol.\% in the X-band. 


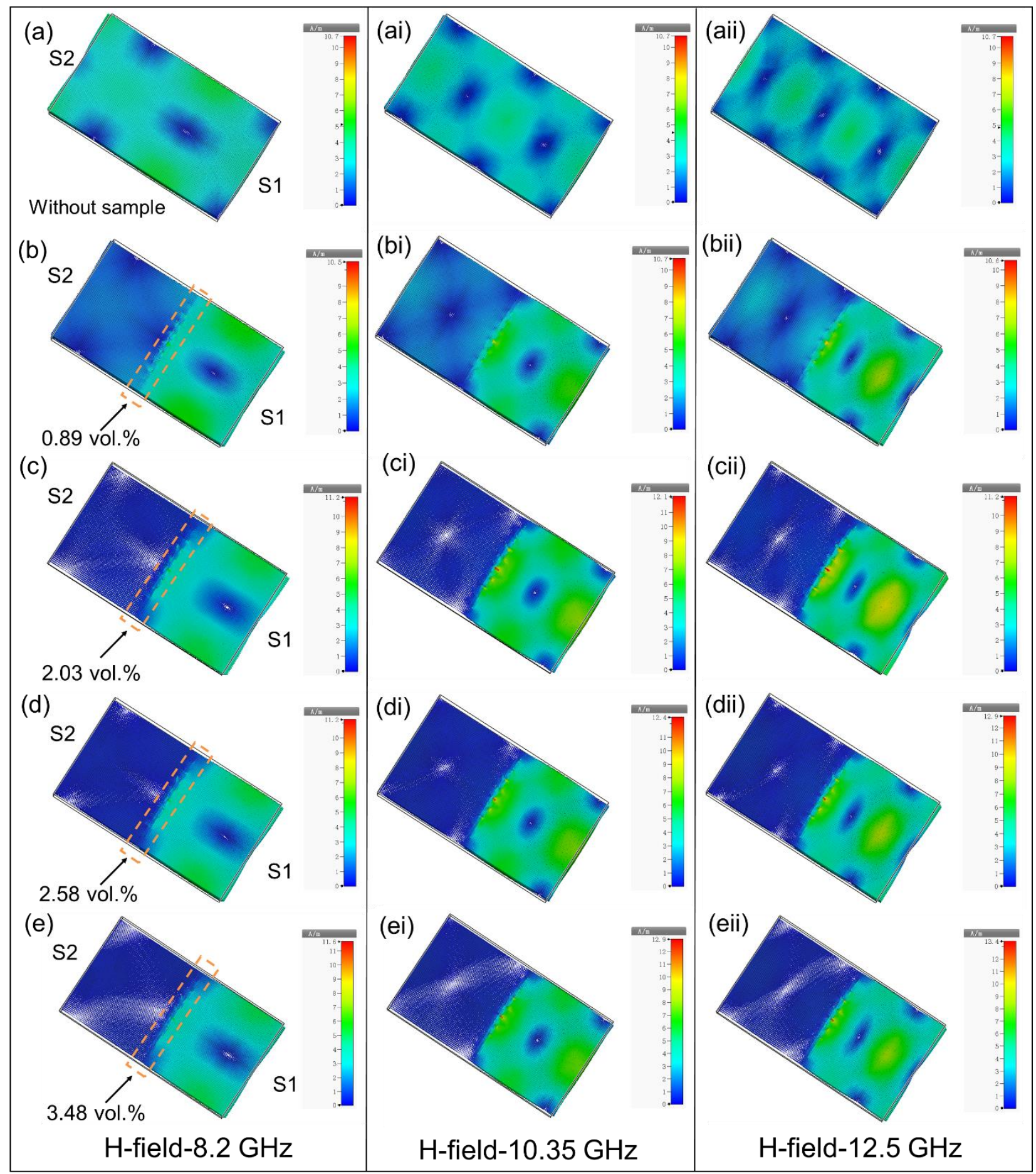

Figure S8. Electromagnetic simulation results obtained by software. Magnetic field intensity distribution of (a) without sample, (b) Ag@ME-0.89vol.\%, (c) Ag@ME-2.03 vol.\%, (d) Ag@ME2.58 vol.\% and (e) Ag@ME-3.48 vol.\% in the X-band. 


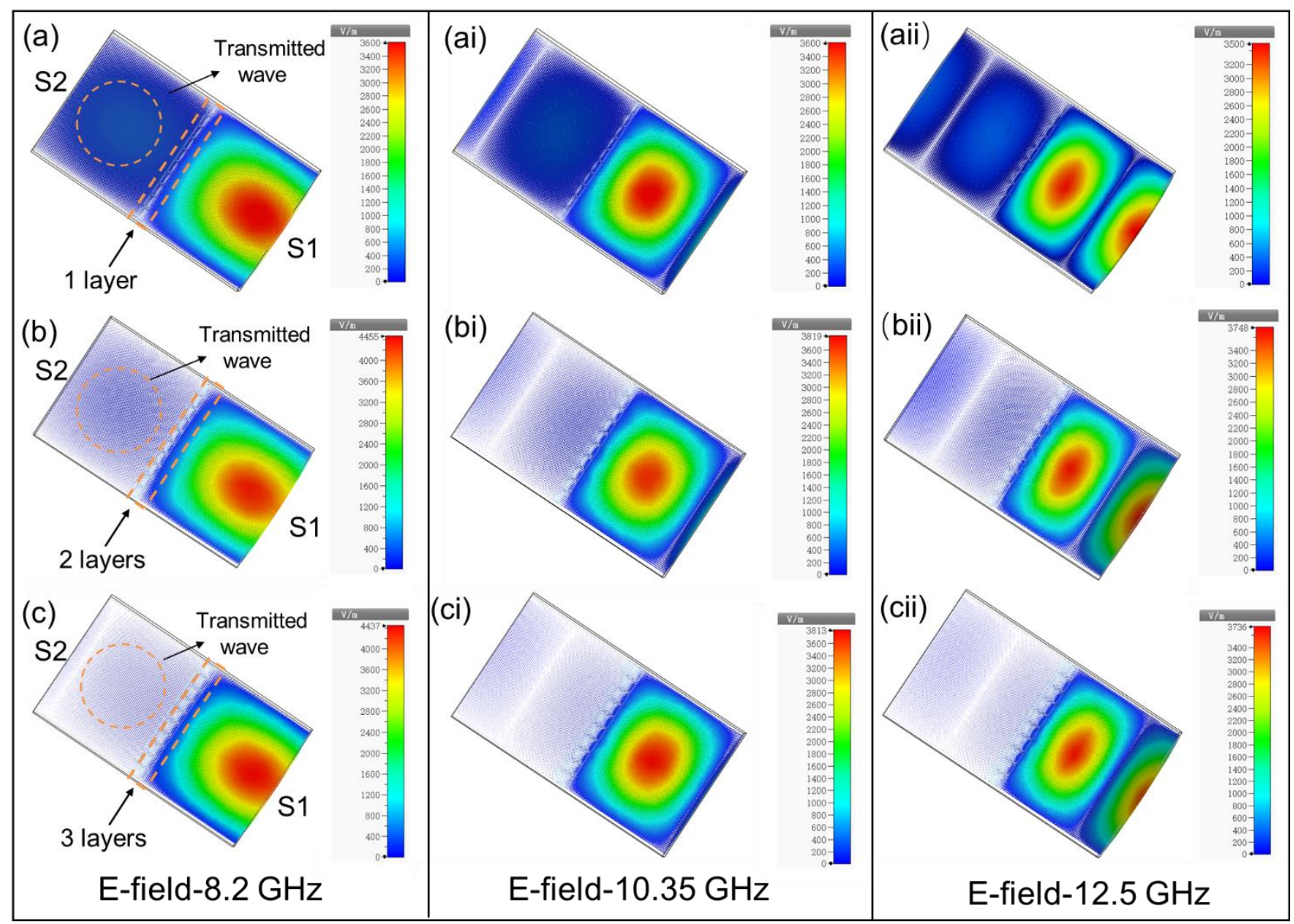

Figure S9. Electromagnetic simulation results obtained by software. Electric field intensity distribution of different layers of (a) 1 layer, (b) 2 layers and (c) 3 layers of Ag@ME-2.03 vol.\% in the X-band. 


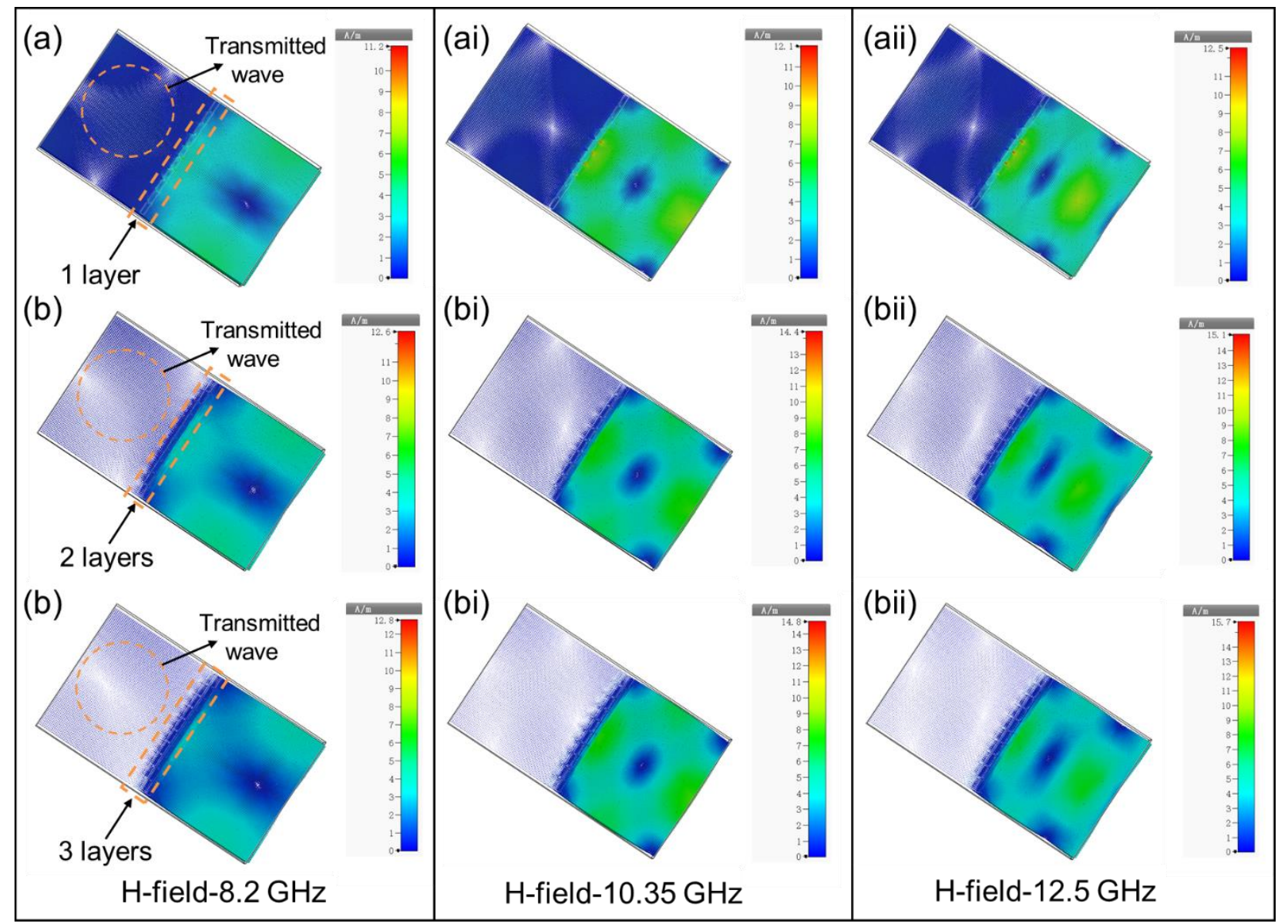

Figure S10. Electromagnetic simulation results obtained by software. Magnetic field intensity distribution of different layers of (a) 1 layer, (b) 2 layers and (c) 3 layers of Ag@ME-2.03 vol.\% in the X-band. 

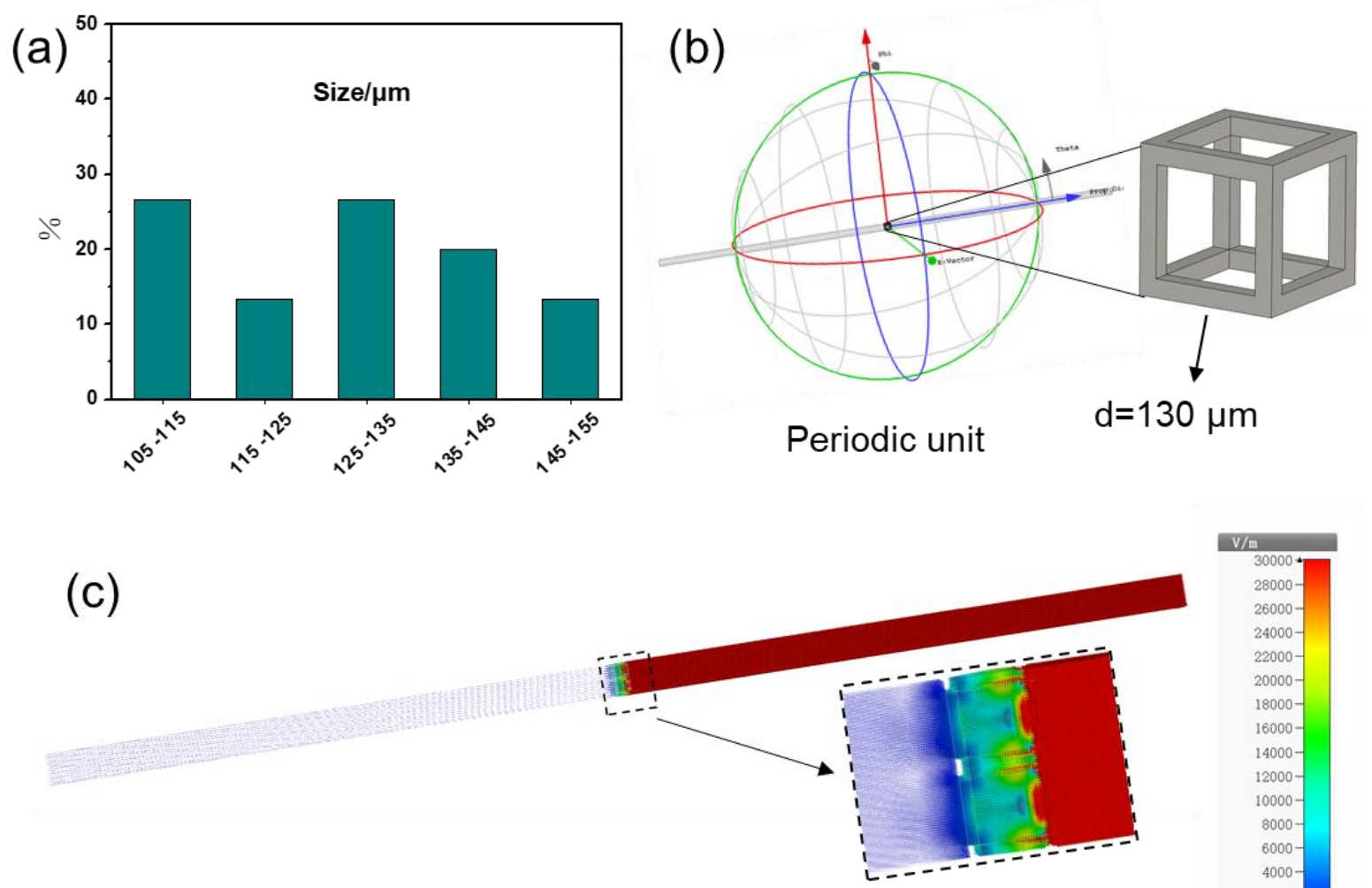

(d)

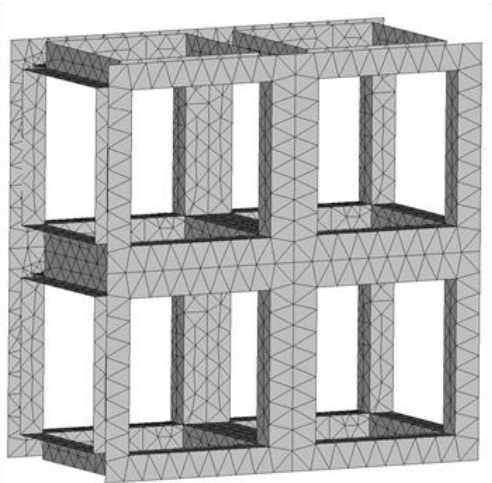

(e)

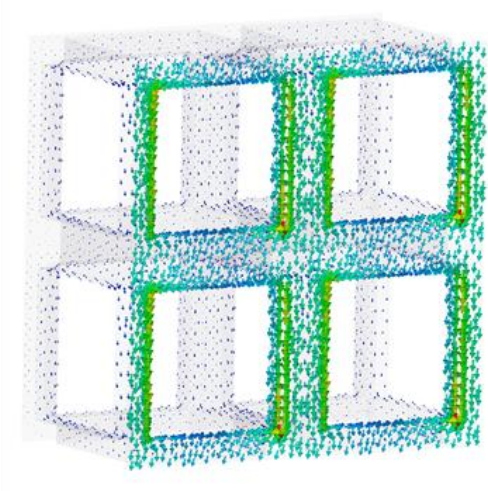

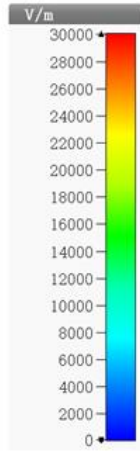

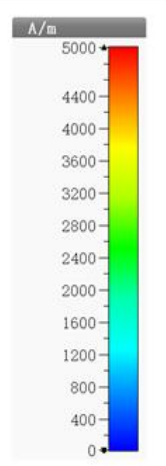

Figure S11. Periodic structure solution and simulation results of Ag@ME-2.03 vol.\% obtained by software. (a) Statistics of pore size distribution of ME from SEM images. (b) Periodic unit size used in modeling. (c) Periodic structures model. (d) Mesh of model. (e) Intensity distribution of surface current. 


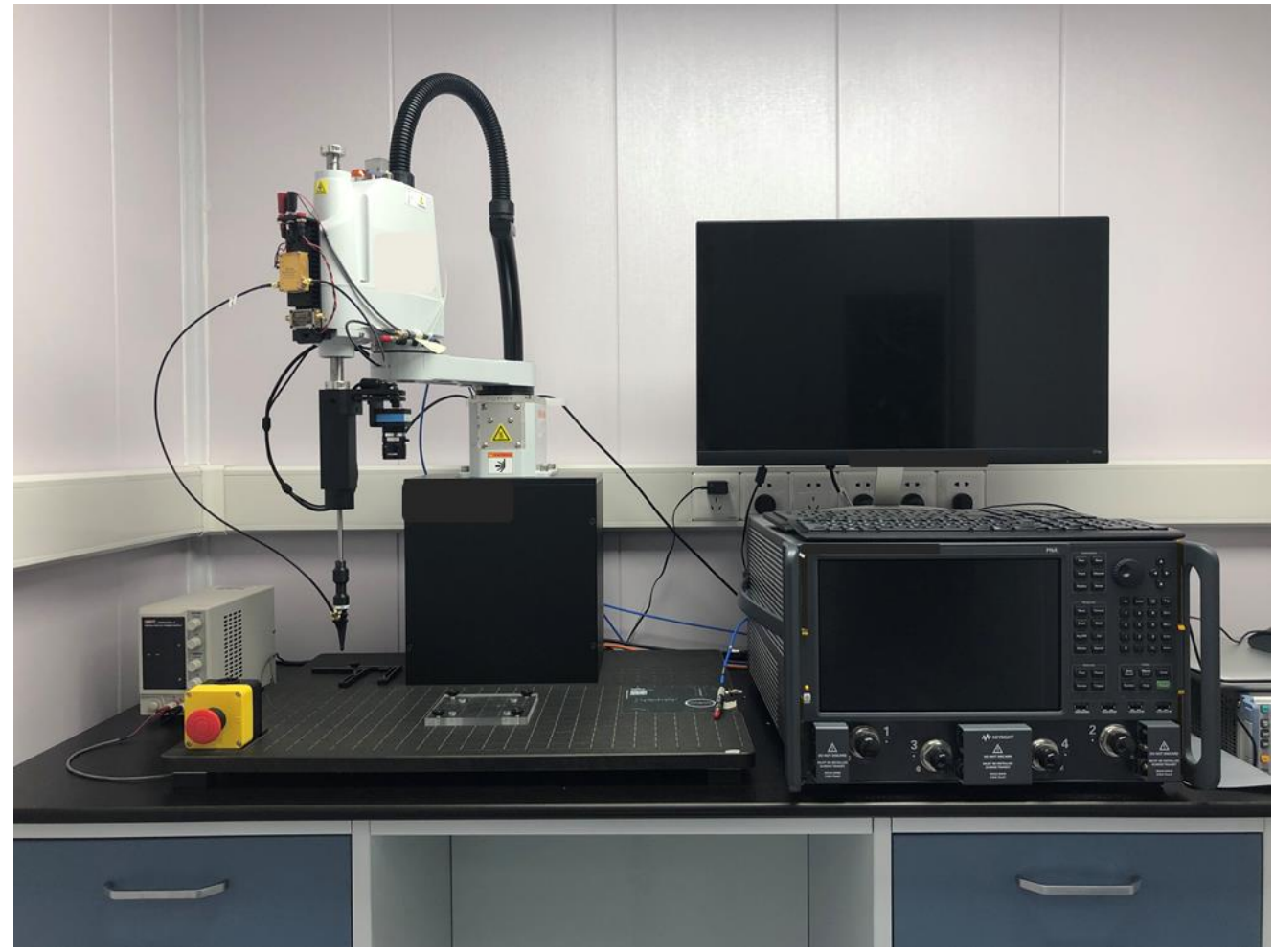

Figure S12. Measurement setup of near-field shielding performance. 